\title{
Novel Process Methodology for Uniformly Cutting Nanotubes
}

Steven R. Lustig*, Edward D. Boyes, Roger H. French, Timothy D. Gierke, Mark A. Harmer, Paula B. Hietpas, Anand Jagota, Greg P. Mitchell, G. Bibiana Onoa and Kerry D. Sams,

Central Research and Development, E.I. du Pont de Nemours \& Co. Inc., Wilmington, DE 19880 U.S.A.

\begin{abstract}
We present a novel process methodology for the controlled cutting of nanotubes and other nanostructures to well-controlled lengths and sizes. The continuing increase in complexity of electronic devices, coupled with decreasing size of individual elements, are placing more stringent demands on the resolution and accuracy of fabrication patterns. The ability to fabricate on a nanometer scale guarantees a continuation in miniaturization of functional devices.

Particularly interesting is the application of nanotubes' chemical and electronic properties which vary with their dimensions and structure. One realization of this process includes the use of photolithography or electron beam lithography to place protective resist patterns over the nanostructures to be cut. Those sections which are not covered by the resist pattern are removed by reactive ion etching. This is a scaleable process which permits the simultaneous cutting of many nanostructures and ensembles of nanostructures. The lengths, shapes or length distributions can be predicted from theory and thus specified for a given application requirement. Nanostructures which can be cut in this process include nanotubes, nanofibers and nanoplanes. Large scale production of nanostructures with uniform length or specific size-distribution can be used in electronic applications such as field-emission transistors, optoelectronic elements, single electron devices and sensors.
\end{abstract}

\section{INTRODUCTION}

Increasingly more stringent demands are placed on the resolution and accuracy of fabricated components to make integrated microdevices more functional and compact. The ability to synthesize and modify components on a nanometer scale guarantees a continuation in miniaturization of functional microdevices, e.g. in electronic circuitry, field emitters, artificial actuators, chemical sensors, energy storages, molecular-filtration membranes, photoabsorbing materials, molecular transistors and other device components. Generalized modification techniques which can trim the shape or length after synthesis are very rare. Particularly interesting is the ability to modify carbon nanotubes, CNTs. CNTs have a diameter in the order of tens of angstroms but are typically synthesized to lengths of several micrometers. Although CNTs exhibit a range of very interesting and useful properties for integrated devices, their long lengths as synthesized present a challenge for application in miniaturized devices. Some reported methods to cut specifically CNTs provide very little control over the cutting precision and protection of the remaining fragments, e.g. acid treatment with ultrasonication [1], microtoming [2], ion bombardment [3] and grinding [4]. A more laborious method [5] is specific to modifying one CNT at a time, which is not readily amenable to mass production of precise structures with uniform size distributions. Other chemical methods [6] cleave random fragments a produce broad length distributions. We believe that none of the prior methods would be economically feasible for bulk quantity synthesis of controlled, sub-micron length CNTs in commodity electronic 
devices. Here we describe a novel lithographic methodology which can cut CNTs precisely and uniformly without further damage. The method is applicable to many other nanostructured materials where one wishes to trim the shape or size as well as modify the peripheral chemical group functionality at the cutting surface.

\section{EXPERIMENT}

The cutting process employs standard microlithography technology [7] coupled with reactive ion etching. By way of example, the cutting process steps for CNTs are described herein and illustrated in Figure 1. The method proceeds by (Fig. 1-1) dispersing CNTs in a solution which enables (Fig. 1-2) spreading the CNTs on the surface of a suitable solid substrate, such as a standard silicon wafer. This layer is then (Fig. 1-3) covered with a photoresist polymer, over which a photomask is applied. Light emanating through the photomask provides a spatial pattern, e.g. long, narrow parallel lines, of photochemical reaction which will define which photoresist areas will be removed or preserved when (Fig. 1-4) the photoresist is developed. After the photoresist is developed, a portion of the CNT population is exposed on the substrate according to the photomask pattern. The exposed CNTs and remaining resist pattern are (Fig. 15) irradiated with oxygen plasma which obliterates only the exposed CNT portions and erodes away some of the resist pattern. Note that portions of CNTs underneath the resist pattern remain protected from etching and damage. At this point only the CNT ends remain exposed at the cutting planes and can be subject to chemical modification or functionalization. In the final step (Fig. 1-6) we dissolve away the remaining resist pattern and resuspend the cut CNTs in a convenient solvent.

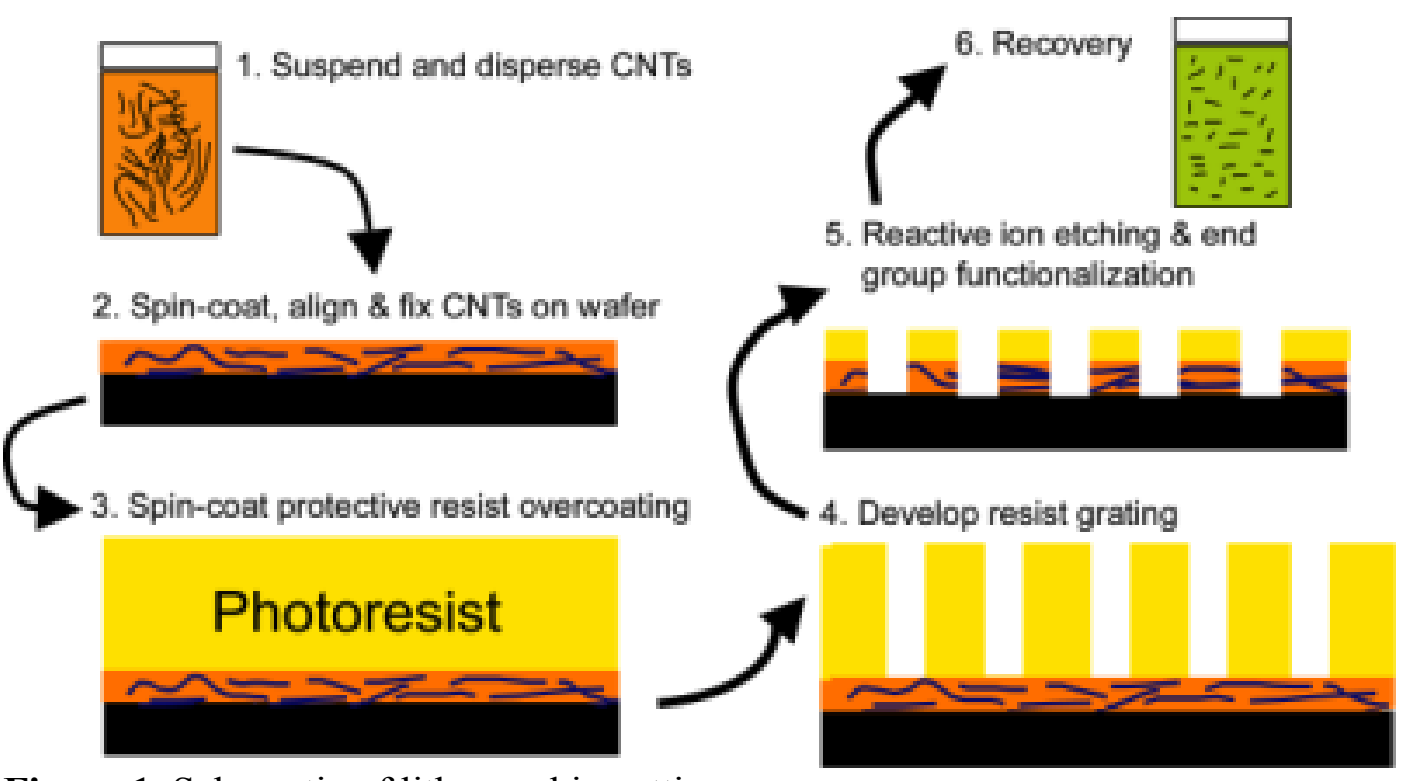

Figure 1. Schematic of lithographic cutting process.

The scanning electron micrographs in Figure 2 illustrate the key facets of the sample preparation and synthesis. The micrograph in Figure 2A is a typical random mat formed by spin coating a $0.3 \mathrm{wt} \%$ dispersion of purified HIPCO single walled carbon nanotubes (SWNTs) in 1:1 chloroform: dichlorobenzene on a 4-inch silicon wafer at 100rpm. Figure 2B exhibits a control silicon wafer with a linear grating of negative photoresist. Shipley UVN30 DUV negative resist 
was spun on the wafer at $3000 \mathrm{rpm}$ and baked for $60 \mathrm{sec}$ at $100^{\circ} \mathrm{C}$. The coated wafer was exposed through a standard resolution pattern photomask with $248 \mathrm{~nm}$ light at an intensity of 28 $\mathrm{mJ} / \mathrm{cm}^{2}$ using a Nikon NSR-1505 EX-1 DUV $248 \mathrm{~nm}$ excimer laser stepper. The wafer was next baked at $100^{\circ} \mathrm{C}$ for $60 \mathrm{sec}$ and developed in AZ300 MIF for $30 \mathrm{sec}$, rinsed in water and dried. The resulting pattern provides photoresist covering in $800 \mathrm{~nm}$ wide protection lines and $400 \mathrm{~nm}$ wide cutting lines. Here, the narrower dark lines are the cutting channels exposing the substrate. The wider elevated light-gray lines are elevated strips of protective photoresist. Figures $2 \mathrm{C}$ and $2 \mathrm{D}$ are sample wafers containing a bottom spun coat SWNT mat and a top positive photoresist patterned identically to the Figure $2 \mathrm{~B}$ control, although now the exposed nanotubes were cut by 90W oxygen plasma at $30 \mathrm{mTorr}$ in an Applied Materials reactive ion etcher. Micrograph 2C shows the effect of $60 \mathrm{sec}$ exposure to oxygen plasma. Note that several uncut SWNT fragments remain exposed in the narrow channels while the protected SWNT segments are visible through the resist by secondary electron emission within the SEM. The sample shown in Figure 2D shows that the SWNTs have been completely cut after $90 \mathrm{sec}$ exposure to oxygen plasma.
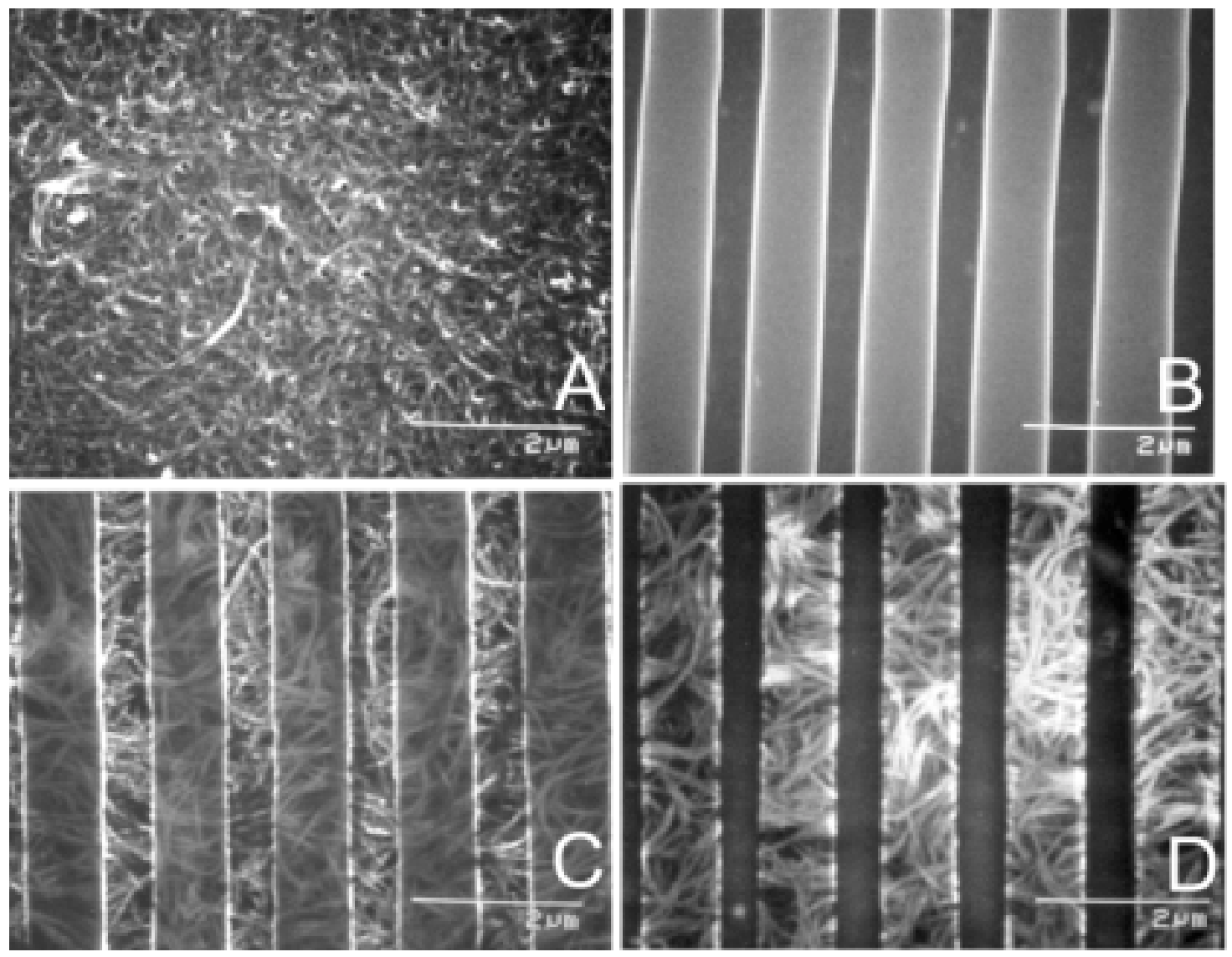

Figure 2. Scanning electron micrographs of key cutting steps.

\section{THEORY}

Even randomly oriented nanotubes are cut into very narrow length distributions using a linear grating photoresist. Figure 3A illustrates cut length probability distributions predicted by geometric Monte Carlo computer simulation. The simulation samples random orientation angles between the photoresist lines and the CNTs as well as the random offsets between the CNT ends 
and the photoresist grating lines. For each statistical sample the simulation itemizes how many sections are cut by a fixed grating and computes the resulting contributions to the length probability distribution. As long as the resist line width, $d$, is much smaller than the original nanotube length, $L$, we note that the predicted length probability distribution is very sharply peaked at the photoresist width. Furthermore, the distribution width and shape are invariant on the logarithmic abscissa. Hence, the distribution width is sensibly independent of the photoresist width.
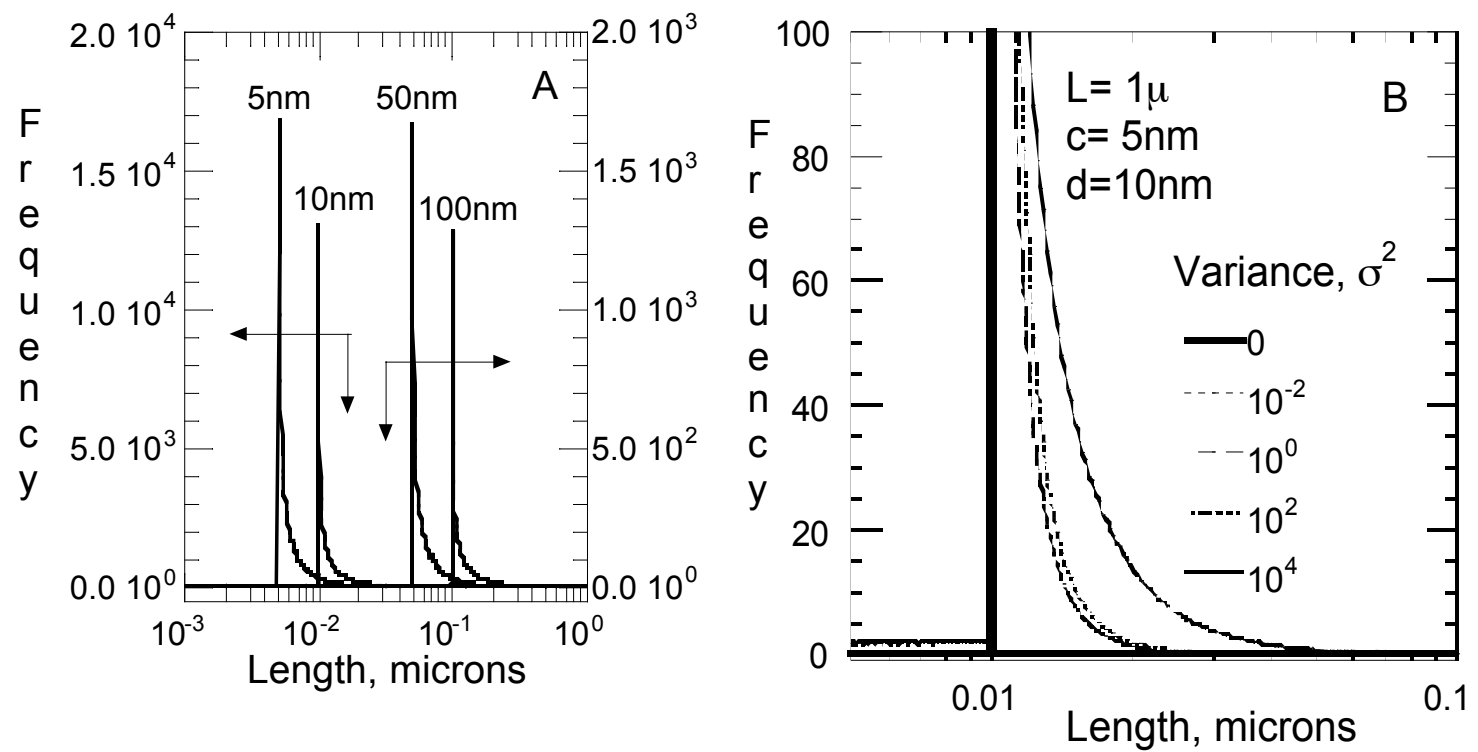

Figure 3. Predicted length probability distribution for CNTs cut with linear grating photomask. (A) CNTs with initial length $L=1 \mu \mathrm{m}$ with random orientation and $c=5 \mathrm{~nm}$ cutting lines. Distributions are shown for resist lines of: $d=5 \mathrm{~nm}, 10 \mathrm{~nm}$ (left), 50nm, 100nm (right). (B) Distribution widths for various degrees of CNT orientational variance normal to the linear grating.

Furthermore, we estimate that even modest degrees of orientation can significantly sharpen the length distribution. Consider the following Gaussian orientation distribution function

$$
P\left(\sigma^{2}, \theta\right)=\frac{8}{\pi(4-\pi)} \sum_{n=1}^{\infty} \frac{(-1)^{n}}{2 n+1} \cos (2 \mathrm{n} \theta) \exp \left(-4 \mathrm{n}^{2} \sigma^{2}\right)+\frac{2}{\pi}
$$

where ${ }^{\theta}$ is the orientation angle between the CNT and the photoresist grating normal and $\mathcal{J}^{2}$ is the orientational variance. Variance as high as $100 \mathrm{deg}^{2}$ provides a broad distribution of orientations while a significant population of nanotubes is perpendicular to the photoresist grating. A variance as low as $10^{4} \mathrm{deg}^{2}$ provides essentially a uniform distribution of orientations in the substrate plane. Figure 3B presents cut length probability distributions predicted by geometric Monte Carlo computer simulation for different degrees of orientational variance. An orientational variance as high as $100 \mathrm{deg}^{2}$ still results in a length distribution where $c a .90 \%$ of the cut CNTs are within $c a .17 \%$ of the protection line width. Hence, even modest efforts to align the CNTs would productively narrow the length distribution.

Simple geometric arguments provide an approximate, analytic expression for the length probability distribution, $\mathrm{g}(l)$, for randomly-oriented CNTs. Consider cutting CNTs that are long compared to cut width, $c$, and resist width, $d$, and whose curvature can be neglected over the 
same length scale. For this simple argument let us neglect misregistry of the CNT ends with the photoresist lines. An individual tube of original length, $L$, may lie at an angle, $\theta$, with respect to the orientation of the resist lines. In order for a CNT to be cut at least once, the CNT orientation angle must exceed $\theta_{\text {min }}$

$$
\sin \theta_{\min }=\frac{d}{L}
$$

A CNT with orientation angle exceeding $\theta_{\min }$ is cut into $n_{\theta}$ pieces

$$
\mathrm{n}_{\theta}=\left\lfloor\frac{L \sin \theta}{c+d}\right\rfloor
$$

with each piece having length, $l$,

$$
l=\frac{d}{\sin \theta}
$$

for $l \geq d$. Let $\mathrm{g}(l) \mathrm{d} l$ equal the number of cut tubes having length between $l$ and $l+\mathrm{d} l$, and let $\mathrm{f}_{\theta} \mathrm{d} \theta$ equal the probability of a tube having orientation between $\theta$ and $\theta+d \theta$. Therefore,

$$
\mathrm{g}(l) \mathrm{d} l=\mathrm{n}_{\theta} \mathrm{f}_{\theta}(-\mathrm{d} \theta)=\mathrm{n}_{\theta} \mathrm{f}_{\theta}\left(\frac{-\mathrm{d} \theta}{\mathrm{d} l}\right) \mathrm{d} l
$$

For randomly oriented nanotubes, $f_{\theta}$ is simply $2 / \pi$. By combining Eqs (3)-(5) we find the probability density distribution, $\mathrm{g}(l)$, is readily obtained for randomly oriented CNTs as

$$
\mathrm{g}(l)=\mathrm{n}_{\theta} \mathrm{f}_{\theta}\left(\frac{-\mathrm{d} \theta}{\mathrm{d} l}\right)=\left\lfloor\frac{L}{c+d} \frac{d}{l} \mid \frac{2 d}{\pi l} \frac{d}{\sqrt{2-d^{2}}}\right.
$$

for $l \geq d$. This distribution is sharply peaked at $l=d$; indeed, the theoretical expression (6) has a square-root, integrable singularity. For $l<d, \mathrm{~g}(l)$ is uniformly $d / \mathrm{L}$, which is due to the tube ends. Any non-uniform orientation of the tubes normal to the cutting lines sharpens the distribution further. Given an orientation distribution, the resulting distribution of length is easily predicted using equation (6), or the more accurate geometric Monte Carlo analysis presented above. In essence, those nanotubes originally oriented nearly normal to the resist lines contribute several fragments to the length distribution, while nanotubes originally oriented nearly parallel to the resist lines contribute far fewer fragments.

\section{RESULTS}

Randomly oriented SWNTs cut from this process do indeed exhibit our expected narrow length distribution. In one recent example randomly oriented SWNTs were cut using 285nm photoresist line widths. The protective photoresist lines were removed, leaving a significant number of bare, cut nanotube fragments adhered to the silicon wafer substrate. Seven random SEM images of the substrate were recorded where cut fragments were visible. Tube length probability distributions were recorded by graphical image analysis of 370 tube bundles using their apparent, relative bundle width as a statistical weight. All tube sections were noted to be straight. Linear sections are expected since the $285 \mathrm{~nm}$ photoresist width is much shorter than the Kuhn segment length of $c a$. 1.6 microns for single wall carbon nanotubes [8]. The resulting length data were collected into 20 statistical bins and resulting probability distribution was normalized to unit area. Figure 4 compares these data to the simple theoretical result in Eq.6 given the same number and width of statistical bins. Theory and experiment are in very good agreement. In 
particular the predicted skewed nature of the length distribution and its width are captured well. Discrepancy between the measured and predicted lengths is greatest at lengths just less than the photoresist width. In these preliminary efforts the resist width varied roughly $5-10 \%$ along each resist line, see Figure 2. We further note that the photoresist lines are reduced in width as well as in height during the oxygen plasma exposure. Better lithographic control of these line widths would produce an even narrower observed probability distribution.

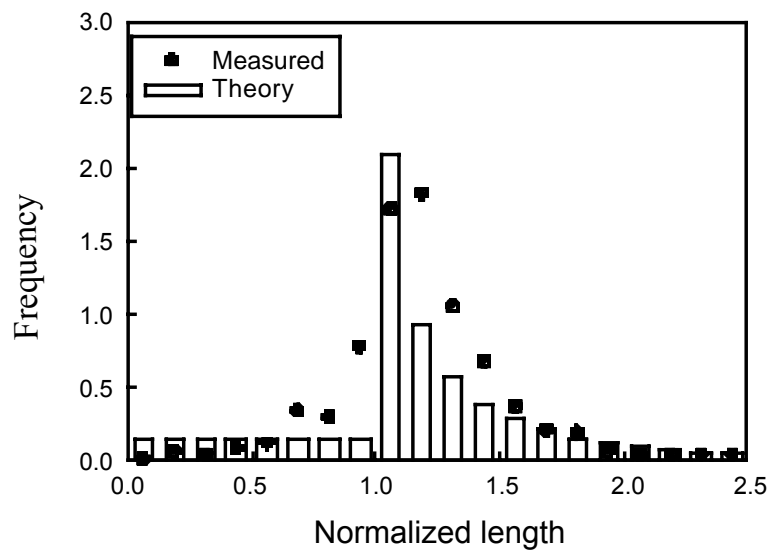

Figure 4. Comparison between the theoretical (Eq. 6) and observed length probability distributions, probability as a function of normalized length $(l / d)$.

\section{ACKNOWLEDGEMENTS}

This work was funded by DuPont Apex program No.749. This work was performed in part at the Cornell Nanofabrication Facility (a member of the National Nanofabrication Users Network) which is supported by the National Science Foundation under Grant ECS-9731293, its users, Cornell University and Industrial Affiliates.

Correspondence should be addressed to S.R.L. (Email at the time of writing this manuscript: steve.r.lustig@usa.dupont.com)

\section{REFERENCES}

1. M. Yudasaka, M. Zhang, C. Jabs, S. Iijima, Appl. Phys. 71, 449 (2000).

2. P.M. Ajayan, O. Stephan, C. Colliex, D. Trauth, Science 265, 1212 (1994).

3. R. Anri, Kokai patent application HEI 7[1995]-172807.

4. I. Stepanek, G. Maurin, P. Bernier, J. Gavillet, A. Loiseau, R. Edwards, O. Jaschinski, Chem. Phys. Lett. 331, 125 (2000).

5. N. Yoneya, E. Watanabe, K. Tsukagoshi, Y. Aoyagi, Appl. Phys. Lett. 79, 1465 (2001).

6. Z. Gu, H. Peng, R.H. Hauge, R.E. Smalley, J.L. Smalley, Nano Lett. 2, 1009 (2002).

7. W. M. Moreau, Semiconductor Lithography: Principles and Materials (Plenum, New York, 1988).

8. M. Sano, A. Karnino, J. Okamura, S. Shinkai, Science 293, 1299 (2001). 